\section{Tomorrow's world}

\section{Jonathan Knight takes a look at what the future may hold for innovation in San Diego.}

$\mathbf{P}$ redicting the future is a notoriously precarious occupation. But San Diego, with its wealth of biotech and high-tech expertise, offers a ready guide to likely sucesses. True, there are a lot of projects that sit firmly in the speculative category, but there are also emerging technologies that are already making their influence felt. Drawing up a comprehensive list of such developments is impossible, but from the marriage of microfabrication with cell biology to a mixture of wireless communication and medical health, a feel for the region's innovation is easy to obtain. Here, in no particular order, we present five of the hot prospects that are helping San Diego to assert itself on the global stage.

\section{Chemical sensors}

What's that smell? Is it toxic discharge from the factory up the river? Is it nerve gas from a terrorist attack? Or is it just an open rubbish bin? Increasingly, people - particularly military personnel and emergency response teams - would like to know what is in the air.

Seacoast Science based in Carlsbad is one of a handful of companies nationwide working on the next generation of accurate, lightweight chemical sensors. It has a prototype that it hopes will end up clipped to the shirts of US Army soldiers in two years' time.

The portability of the chemical detectors currently used by the US military is hampered by the fact that they are powered by batteries weighing several kilograms. In addition, the sensors are not particularly discerning. An alarm could mean a nerve-gas attack or that farmers down the road are spraying pesticides on their fields.

Part of a solution to the portability problem is to use microelectromechanical systems: fingernail-sized devices that require only 5 microwatts for one chip of ten capacitors. Seacoast's design packs ten sensors onto a chip just $2 \mathrm{~mm}$ by $5 \mathrm{~mm}$. Each sensor is 360 micrometres in diameter and consists of a thin layer of specialized polymer sandwiched between two conductive discs. The chemical of interest enters the sensor through perforations in the top disc and is absorbed by the polymer, altering the electrical capacitance of the polymer sandwich. This change is detected electronically.

The polymer layer is the key to this technology. Todd Mlsna, Seacoast's co-founder and president, has developed a series of absorbent polymers, each sensitive to a different class of chemical. Each of the ten sensors on a chip contains a different polymer, and any given gas changes the capacitance of the polymer sandwich at a different rate. This produces a unique electronic signature for the gas, and enables the chip to distinguish far more than ten compounds. Byvarying the polymers, Seacoast can tailor a chip to a given class of chemical.
In October, the company tested its sensor at the US Army Medical Research Institute of Chemical Defense in Aberdeen Proving Ground, Maryland, and showed that it could detect minute concentrations of nerve gas. Seacoast has already produced six prototypes for the Marines and is now developing ones with sensitivity to toxic industrial chemicals.

The advantage of Seacoast's sensors is their portability - every soldier can carry one. The chips do not yet distinguish nerve agents from chemically related pesticides, but this may be possible as more sensors are developed or a chemical-separation step added, according to Mlsna. "We like to think we are developing the heart of all future sensor systems," he says.

\section{Artificial livers}

Could making artificial organs one day be as simple as assembling computer chips? Sangeeta Bhatia, a bioengineer at the University of California, San Diego, thinks

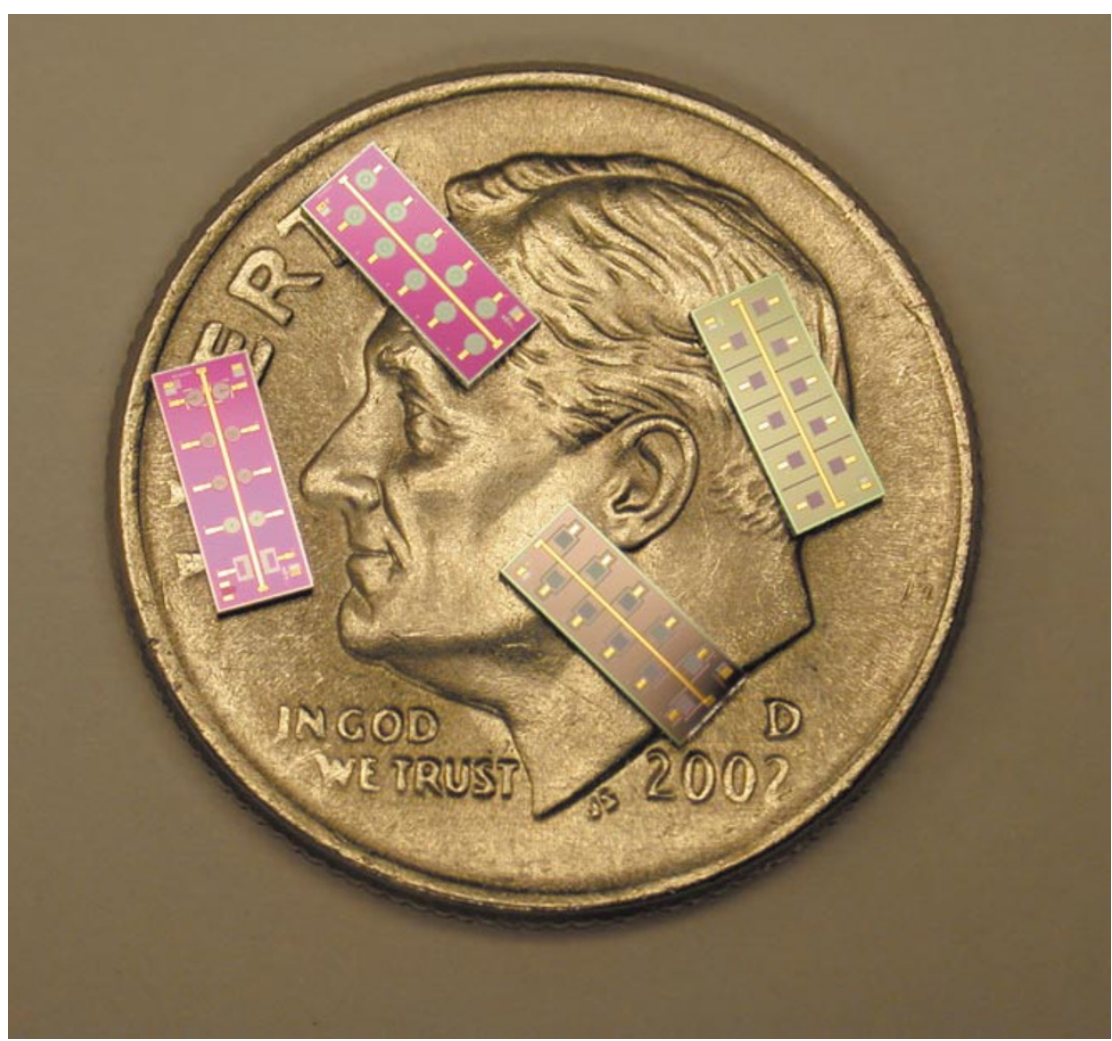

Small and sensitive: Seacoast's chemical sensors can distinguish between several gases. 


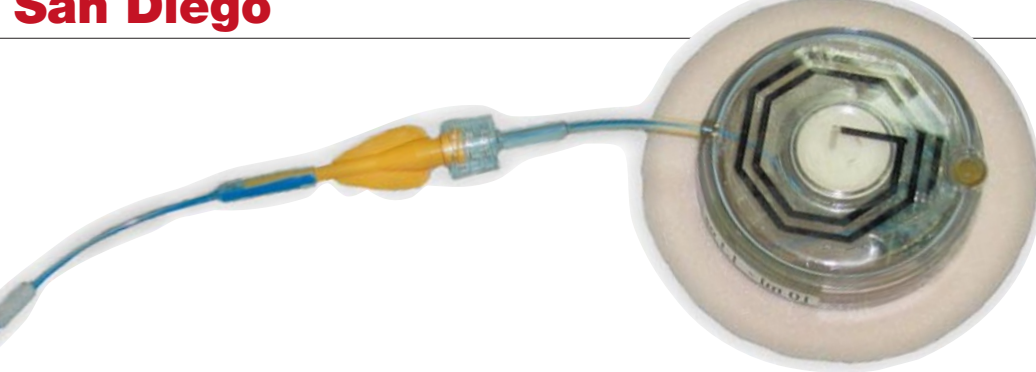

Control and construction: PhiloMetron's smart medicine patch monitors the heart and sends this information to the doctor, who can then decide how much medication to dispense (left). A three-dimensional liver-like tissue structure made from three layers of liver cells. Each layer of cells is fluorescently labelled with a different colour dye (below).

so. She is borrowing tools from the semiconductor industry to build artificial livers. So far, she has made only tiny liver fragments, but she hopes one day to build an entire lobe of liver suitable for transplanting into a patient.

Transplants are currently the only cure for patients with severe liver damage. But there aren't enough livers being donated. In the United States alone, 18,000 patients are waiting for a liver transplant, according to the American Liver Foundation, outstripping supply by fourfold.

External artificial livers, which are similar to kidney dialysis machines except that they use living cells, would extend the lives of patients waiting for a donor, but previous attempts to develop these have failed in clinical trials. One reason for this, Bhatia says, is that the liver cells tend to lose their ability to detoxify blood. To solve this problem, she grows the liver cells next to the support cells that surround them in the real organ and provide essential growth factors. She uses microfabrication tools developed for making microchips in Silicon Valley to lay down the cells in specific patterns and so control how much they interact with one another. This produces an artificial liver that is ten times more effective compared with one based on liver cells alone, she says.

But to make an entire liver suitable for transplantation, it is necessary to move away from two-dimensional cell cultures because livers are three-dimensional, not merely a layer of cells. Bhatia has begun making three-dimensional liver sections using another microfabrication method called stereolithography. This techniques uses a liquid resin that is solidified layer by layer by a laser beam. For example, to make an object shaped like a tumbler, the beam sweeps back and forth across a shallow dish of resin until it forms a solid disk, which will be the bottom of the tumbler. The disk is submerged slightly deeper in the resin so that the next layer can be solidified. The laser beam must hit the liquid resin — if the disk is floating the beam will only hit the already hardened disk. Further successive rings make up the sides of tumbler. This technique can be used to make almost any three-dimensional structure.

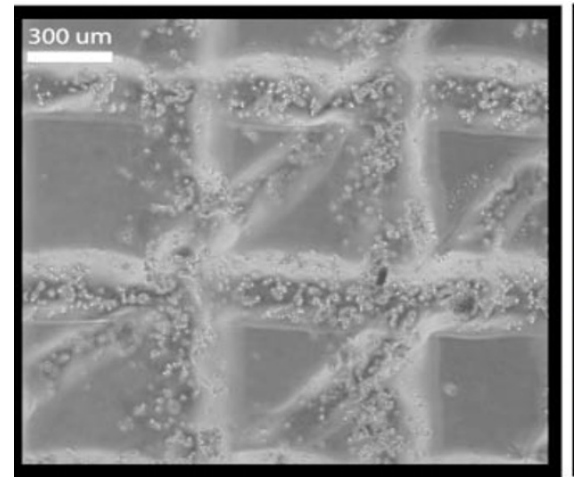

Bhatia has developed a resin that will support growing cells (V. A. Liu and S. N. Bhatia Biomed. Microdev. 4, 257-266; 2002). She mixes in liver cells, and then builds up successive layers of cells into a cube with sides of $1 \mathrm{~mm}$. She leaves holes in each layer so that this small section of artificial liver has a branching vasculature like that found in real liver tissue. Bhatia uses stereolithography to make tiny liver chunks, in which the cells survive and are supported by the matrix. Work is under way to test their function.

The first application for these artificial livers could be in testing new drugs. Some $25 \%$ of new drugs fail clinical trials on people because the liver inactivates them too quickly. If this could be anticipated, the pharmaceutical industry could save a great deal of money.

Making a functional liver for transplant is still far in the future, Bhatia says. At the moment she is using just one type of support cell, but at least three of the five major cell types found in the liver would be needed for a functional organ. This requires a much deeper understanding of how the cells interact.

\section{Medicine patches}

Only about half the patients receiving prescription medications for a chronic illness actually take them. For people with a serious condition, such as diabetes or congestive heart failure, non-compliance can mean a trip to the hospital, or worse.

Wearable devices that automatically dispense medications have been sought after for years, but very few are actually on the market.

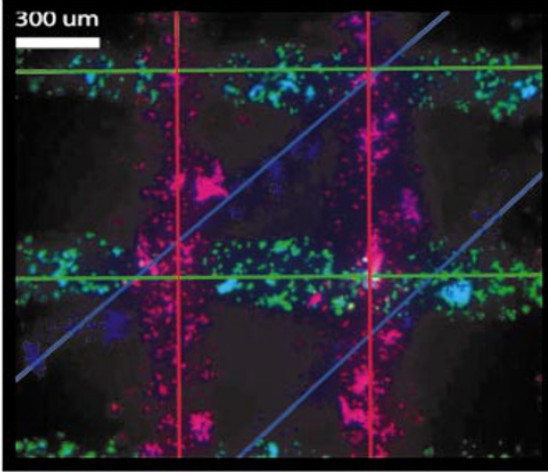

Insulin pumps for diabetics that look like pagers and are worn on a belt are available, but they don't automatically measure blood glucose levels or communicate with the physician who is managing the disease.

PhiloMetron, a start-up company in Sorrento Valley, is now taking the first steps towards tele-electronic medicine dispensers by developing what is essentially a smart skin patch that phones your doctor. The patch, which measures about $5 \mathrm{~cm}$ in diameter, monitors the electrical activity of the heart and sends the data at regular intervals to a nearby mobile phone, which in turn calls the doctor's office; the two steps avoid the need for a bulky transmitter in the patch itself. A clinician looks at the readings, decides how much drug to dispense, and transmits the instructions back to the skin patch, which then delivers the drug. All the patient has to do is change the patch once a week.

PhiloMetron's initial design takes advantage of an existing technology, an implanted cardio defibrillator (ICD), to gather the heart data. This is a sort of super-pacemaker that shocks the heart into a regular rhythm in the event of a heart attack. The patch can potentially go one step further too. By linking to the data provided by the ICD, it could reduce the chance of a heart attack by dispensing drugs at the appropriate time.

The next generation of patches are likely to house the data-gathering information too, so that they will work for patients who don't have an ICD fitted.

For the moment, PhiloMetron, with fewer than ten employees, remains entirely focused on the cardiac application, says president and chief executive Darrel Drinan. But it is not hard to imagine tele-medicine applications for a variety of diseases. Sensors that 
transmit data to the clinic from wherever the patient is would not only cut down on visits to the doctor and the hospital, they would allow physicians to spot problems before they became severe.

During the severe acute respiratory syndrome (SARS) epidemic, in which the first sign of infection was fever, Drinan says he had a number of e-mails from Hong Kong asking if he had anything that could remotely monitor body temperature. "The interest in this concept is extremely high," he says.

\section{Free-space optics}

San Diego is most closely associated with one technology: wireless communication. Wireless transmission usually involves radiowaves or microwaves, but several companies in the San Diego region are developing technology based on something a little more tangible — light.

Fibre-optic cables already use light to transfer huge amounts of data. Thousands of miles of cable have been laid in the past decade, yet relatively few businesses or consumers have fibre-optic links to their desktop computers or between their private networks. This is because of the 'last mile' problem: bringing fibre-optic cables into existing buildings and houses means digging up streets and car parks. As long as existing copper wire - telephone lines and television cables - provide a reasonably fast service, the incentive to replace this technology isn't there.

Lightpointe is one of four major companies - all of which are based on the US west coast - that aim to overcome this issue by ditching the cable altogether. Free-space optics (FSO) is, in effect, fibre optics without the glass. Air is a perfectly good transmission medium for light, so sending a high-bandwidth data stream from one building to the next could be as simple as mounting a laser on one rooftop and pointing it at a receiver on another. Already there are some 10,000 FSO units installed worldwide.

Surprisingly, rain and snow do not affect the light signal. This is because Lightpointe units use four parallel laser beams to help keep the signal continuous. And lost data packets are sent again, as they usually are on the Internet. "It has to be complete white-out conditions to stop these," says Jeff Bean, spokesman for Lightpointe.

But FSO technology does have an
Light work: This unit from Maxima transmits light through air, avoiding the need for wires or cables (top). Computer models from Genomatica help biologists cope with vast amounts of genomic and biochemical data (bottom).
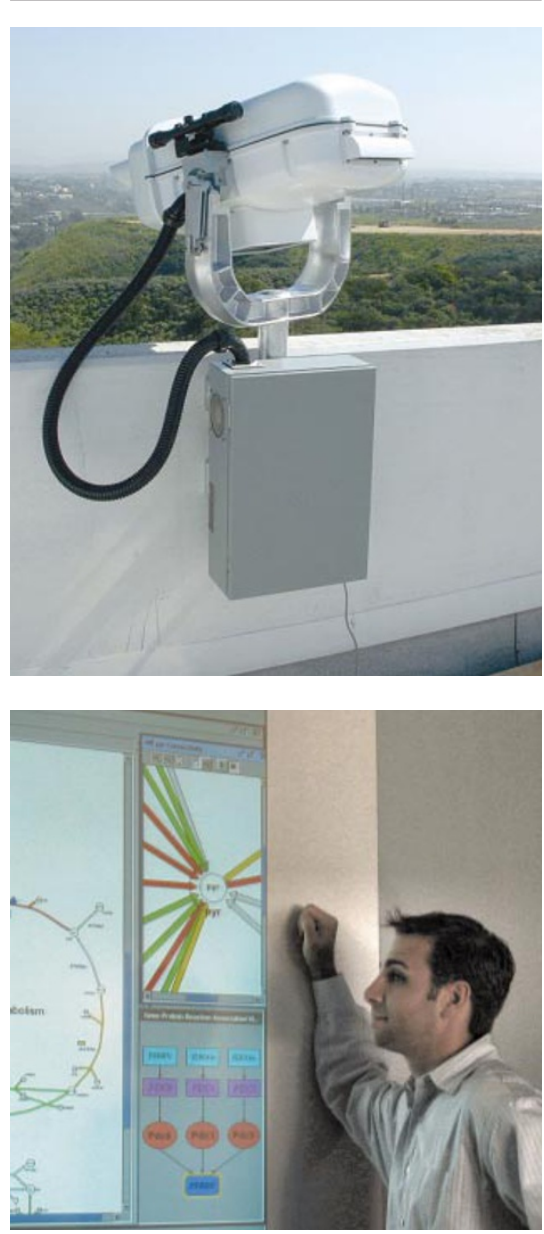

Achilles' heel: fog. The average size of fog droplets is one micrometre, the same as the wavelength used by standard data transmission lasers. Even a light fog can shut down FSO.

Maxima, a company based in Sorrento Valley, is trying to solve this problem by using longer wavelengths of light. Its research suggests that a wavelength of 10 micrometres would be transmitted through fog, but lasers for this wavelength are not widely available. So Maxima has joined forces with the small Swiss company Alpes Lasers, based in Neuchâtel, which has developed a laser smaller than the head of a pin that transmits a 10-micrometre beam with sufficient strength to be read hundreds of metres away. Another company, Vigo Systems in Warsaw, Poland, has designed an appropriate detector, and Maxima plans to try the new units out on customers' rooftops by the end of 2004.

The longer wavelength has other advantages, says president and founder of Maxima James Plante. It is so far in the infrared, that sunlight has very little effect on it, so there is no interference when the Sun sets behind a data link.

\section{In silico biology}

The sequencing of a range of genomes in recent years has left many biologists with a problem. How can they make sense of so much data, particularly when the information relates to thousands of proteins involved in a slew of interdependent biochemical pathways.

Bioinformatics is helping biologists to cope with the reams of data they face every day. But few efforts are aimed at bringing all the data together into a single model to form a digital organism with the power of prediction.

Bernard Palsson, a bioengineer at the University of California, San Diego, has designed computer models of bacteria that incorporate the constraints of all their known metabolic pathways. In a paper in Nature last year, he and his team showed that their approach could accurately predict how a strain of the bacterium Escherichia coli would adapt to a new source of food (R. U. Ibarra, J.S. Edwards and B. O. Palsson Nature 420, 186-189; 2002).

The San Diego company Genomatica, cofounded by Palsson and his former student Christophe Schilling, is now making its computerized bacteria available to researchers worldwide. The model, dubbed SimPheny, incorporates everything known about an organism's physiology, from how it metabolizes food to how it divides.

And the models aren't limited to one type of bacterium. Derek Lovley, a microbiologist at the University of Massachusetts in Amherst who works on the bacterium Geobacter, is a complete convert. In one case, SimPheny helped Lovley to work out which gene was mutated in a strain of Geobacter that was growing poorly in culture. In another, it predicted that a particular mutation would produce a strange metabolic effect that Lovley would not have tested for had the program not come up with it, he says.

Jonathan Knight is a contributing correspondent for Nature. 